\title{
Extensions of the Extrema Bounds for the Pomeron Intercept to Meson-Proton, Gamma-Proton and Gamma-Gamma Scattering
}

\author{
E. G. S. Luna, M. J. Menon, and J. Montanha \\ Instituto de Física Gleb Wataghin, Universidade Estadual de Campinas, 13083-970 Campinas, SP, Brazil
}

Received on 15 August, 2003.

\begin{abstract}
Making use of the extrema bounds for the soft pomeron intercept, recently determined by fits to $p p$ and $\bar{p} p$ data (from both accelerator and cosmic-ray experiments), we investigate the total cross sections for pion-proton, kaon-proton, gamma-proton and gamma-gamma scattering. We show that by means of global fits, the extrema bounds are in agreement with the bulk of experimental data presently available, and that extrapolations to higher energies indicate different behaviors for the rise of the total cross sections. We also discuss factorization and quark counting, showing that both bounds are in agreement with these properties.
\end{abstract}

\section{Introduction}

It is an experimental fact that all hadronic cross sections rise asymptotically with the energy of the collision [1]. In Regge Pole phenomenology [2], the amplitude for a two body scattering at high energies is expressed as a sum of contributions from all the exchanged trajectories allowed in the reaction. By means of the optical theorem,

$$
\sigma_{t o t}(s)=\frac{1}{s} \operatorname{Im} F(s, t=0),
$$

the total cross section is written as

$$
\sigma_{\text {tot }}(s)=\sum_{i} \gamma_{i}(t=0) s^{\alpha_{i}(t=0)-1},
$$

where $\sqrt{s}$ is the c.m energy, $t$ is the four momentum transfer squared, $\alpha_{i}(t)=\alpha_{0}+\alpha^{\prime} t$ is the Regge trajectory related with the exchange of a reggeon carrying the appropriated quantum numbers for the reaction, and $\gamma_{i}$ is the reggeon coupling.

At high energies, the total cross section is dominated by a trajectory with the quantum numbers of the vacuum, known as the pomeron. Therefore, in this context, the rise of the hadronic total cross section is due to the pomeron exchange, and to understand the asymptotic behavior of the cross section means to understand the pomeron's properties and parameters. Since there are no known particles related with the pomeron trajectory, it is necessary to perform fits to the available data in order to establish the Pomeron's parameters, namely, its intercept $\alpha_{\mathbb{P}}(t=0)$ and coupling.

However, the experimental behavior of the hadronic total cross section at the highest energies is far from clear. The data available at this extreme region concern $\bar{p} p$ and $p p$ scattering. In the former case, it is well known the discrepancy at $\sqrt{s}=1.8 \mathrm{TeV}$ between the published results by the CDF Collaboration [3] and the E710 and E811 Collaborations $[4,5]$. The $\mathrm{CDF}$ datum is more than $2 \sigma$ higher than those from E710/E811 and, therefore, indicates a faster grow with energy of the $\bar{p} p$ total cross section. In the later case the estimations of $\sigma_{t o t}^{p p}$ from cosmic-ray experiments $(\sqrt{s}=$ $6-40 \mathrm{TeV}$ ) are model dependent and different approaches provide quite different values for the $p p$ total cross section (see [6] for a recent review, discussions and a complete list of references). Recently, the effects of all these discrepancies have been investigated in Ref. [7]. Fits to $p p$ and $\bar{p} p$ data taking into account all the experimental information allowed the determination of extrema bounds for $\alpha_{\mathbb{P}}(t=0)$, and therefore for the raise of the corresponding total cross sections.

In this work we use these extrema bounds for the pomeron intercept in order to extend the analysis to mesonproton, gamma-proton and gamma-gamma scattering and also to perform tests concerning factorization and quark counting. The paper is organized as follows. In Sec. II we briefly recall the determination of the extrema bounds from $p p$ and $\bar{p} p$ scattering. In Sec. III we present the extensions to the other reactions and the tests about factorization and quark counting. The conclusions and some final remarks are the content of Sec. IV.

\section{Extrema bounds from $p p$ and $\bar{p} p$ scattering}

The effects of the experimental discrepancies, referred to in the previous section, have been investigated through different selections of the experimental information available [7]. First, the accelerator data on $p p$ and $\bar{p} p$ scattering were split in two ensembles, where each ensemble displays one of the 
two possible scenarios for the total $\bar{p} p$ cross section behavior with energy:

- Ensemble I - Fastest increase scenario: $\sigma_{t o t}^{p p}$ and $\sigma_{\text {tot }}^{\bar{p} p}$ data $(10 \leq \sqrt{s} \leq 900 \mathrm{GeV})+\mathrm{CDF}$ datum $(\sqrt{s}=1800 \mathrm{GeV})$.

- Ensemble II - Slowest increase scenario: $\sigma_{t o t}^{p p}$ and $\sigma_{\text {tot }}^{\bar{p} p}$ data $(10 \leq \sqrt{s} \leq 900 \mathrm{GeV})+\mathrm{E} 710 / \mathrm{E} 811$ data $(\sqrt{s}=1800 \mathrm{GeV})$.

In a second step, the highest and lowest estimations for $\sigma_{t o t}^{p p}$ from cosmic-ray experiments have been adequately added to the above ensembles. These estimations correspond, respectively, to the results by Nikolaev [8], together with that by Gaisser, Sukhatme and Yodh [9] (NGSY) and the results by Block, Halzen and Stanev (BHS) [10] (BHS) (see [6] for details). These two other ensembles were denoted by

- Ensemble I + NGSY

- Ensemble II + BHS

In this way one may explore the contrasting data and the faster and slower increase scenarios for the rise of the total cross section, allowed by the experimental information available.

The parametrizations used for the total cross section comes from an extended Regge Pole model. The corresponding forward scattering amplitude $F(s, t=0)$, is decomposed in three reggeon exchanges:

$$
F(s)=F_{\mathbb{P}}(s)+F_{a_{2} / f_{2}}(s)+\tau F_{\omega / \rho}(s),
$$

where $F_{\mathbb{P}}$ is the single Pomeron exchange, $F_{a_{2} / f_{2}}$ is the Reggeon with $C=+1, F_{\omega / \rho}$ is the Reggeon with $C=$ -1 , and $\tau=+1(-1)$ for anti particle-particle (particleparticle). Here, the intercepts of the Pomeron and the $C=$ +1 and $C=-1$ trajectories are expressed by:

$$
\begin{aligned}
& \alpha_{\mathbb{P}}(0)=1+\epsilon ; \\
& \alpha_{+}(0)=1-\eta_{+} ; \\
& \alpha_{-}(0)=1-\eta_{-} .
\end{aligned}
$$

With the above amplitude, and through the optical theorem, Eq. 1, the total cross sections for $p p$ and $\bar{p} p$ interactions reads

$$
\sigma_{t o t}(s)=X s^{\epsilon}+Y_{+} s^{-\eta_{+}}+\tau Y_{-} s^{-\eta_{-}},
$$

where $X, Y_{+}, Y_{-}, \epsilon, \eta_{+}$and $\eta_{-}$are parameters to be fixed by the fit to the data.

Besides the four ensembles defined above, the available data on the ratio of the real to the imaginary part of the amplitude, $\rho=\operatorname{Re} F(s, t=0) / \operatorname{Im} F(s, t=0)$, were also used in global fits involving $\rho$ and $\sigma_{\text {tot }}$. This has been done by means of dispertion relations and either using the subtraction constant $K$ as a free fit parameter or assuming $K=$
0. Among the 16 fits performed through the CERN-minuit program, the extrema bounds for the pomeron intercept were obtained in the case of individual fits to $\sigma_{t o t}$, the upper value with ensemble I + NGSY and the lower value with ensemble I. The value of the fit parameters and statistical information are displayed in Table 1, and the corresponding behavior of total the cross sections, as function of the energy, are shown in Fig. 1, together with the experimental information.

TABLE 1. Parameters obtained from fits to $p p$ and $\bar{p} p$ total cross section and corresponding to the highest and lowest values for the pomeron intercept [7].

\begin{tabular}{ccc}
\hline Fit: & \multicolumn{2}{c}{ Individual $-\sigma_{\text {tot }}$} \\
Ensemble: & $\mathrm{I}+$ NGSY & $\mathrm{II}$ \\
\hline$\epsilon$ & $0.104 \pm 0.005$ & $0.085 \pm 0.004$ \\
$X(\mathrm{mb})$ & $16 \pm 1$ & $20 \pm 1$ \\
$\eta_{+}$ & $0.28 \pm 0.03$ & $0.38 \pm 0.04$ \\
$Y_{+}(\mathrm{mb})$ & $51 \pm 4$ & $62 \pm 8$ \\
$\eta_{-}$ & $0.42 \pm 0.04$ & $0.42 \pm 0.04$ \\
$Y_{-}(\mathrm{mb})$ & $17 \pm 4$ & $17 \pm 4$ \\
$K$ & - & - \\
No. DOF & 94 & 89 \\
$\chi^{2} / \mathrm{DOF}$ & 1.01 & 0.94 \\
\hline
\end{tabular}

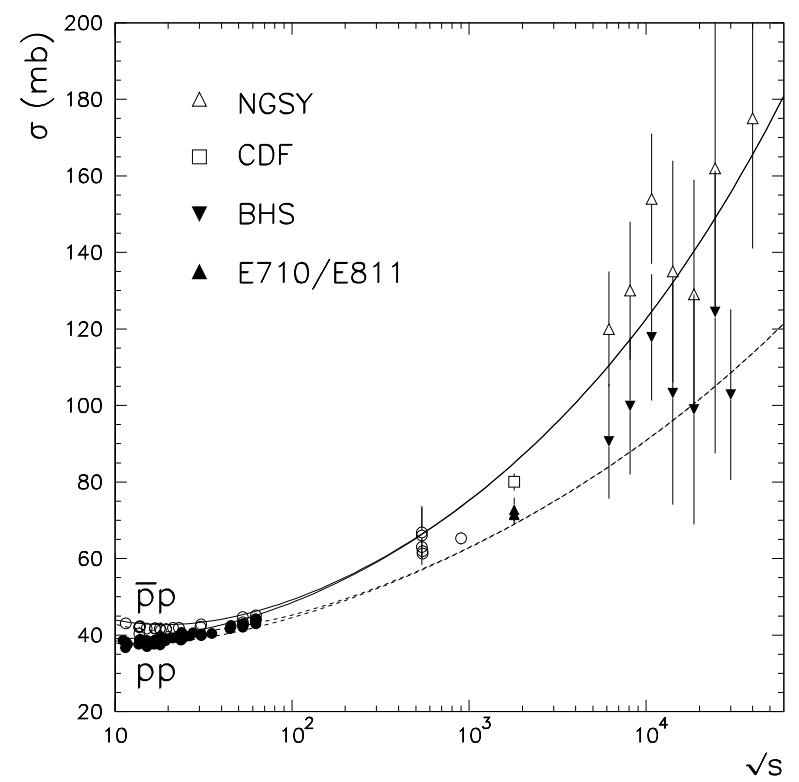

Figure 1. Fastest and slowest increase scenarios for the rise of the total cross section through parametrization (4) and allowed by the experimental information available: fits to ensembles I + NGSY (solid) and II (dashed). See Table 1.

The extrema bounds for the Pomeron intercept are obtained by summing or subtracting the errors to the mean values in Table 1:

$$
\begin{aligned}
& \epsilon_{\text {upper }}=0.0104+0.005=0.109, \text { from I }+ \text { NGSY; } \\
& \epsilon_{\text {lower }}=0.085-0.004=0.081, \text { from II. }
\end{aligned}
$$




\section{Extension to $\pi^{ \pm} p, K^{ \pm} p, \gamma p$ and $\gamma \gamma$ scattering}

Besides $p p$ and $\bar{p} p$, several other hadronic reactions have been measured through the last decades [1]. Although none of them has the energy range of $p p$ and $\bar{p} p$, some reactions have been measured up to considerably high energy values. For meson-proton, the total cross section data for $\pi^{ \pm} p, k^{ \pm} p$ have been recorded up to around $\sqrt{s}=30 \mathrm{GeV}$, whereas for $\gamma p$ and $\gamma \gamma$ there are measurements reaching $200 \mathrm{GeV}$. Therefore, these reactions provide a good ground for investigating the affects of the extrema values obtained for the soft pomeron intercept.

Among other possible procedures for the extensions, we adopt here parametrization (4) and global fits to $\sigma_{t o t}$ data from $\pi^{ \pm} p, K^{ \pm} p, \gamma p$ and $\gamma \gamma$ scattering. We fix the intercepts $\epsilon, \eta_{+}$and $\eta_{-}$, obtained from $p p$ and $\bar{p} p$ scattering, in the cases of the highest and lowest $\epsilon$ values (Table 1). With this procedure we have 10 free parameters: the strengths $X, Y_{+}$ and $Y_{-}$for $\pi^{ \pm} p, K^{ \pm} p$, and $X, Y_{+}$for $\gamma p$ and $\gamma \gamma$ scattering. The total number of data points are 134 and therefore 124 DOF. The fits have been performed through the CERNminuit routine in both cases of the extrema bounds, namely, $\epsilon=0.081$ and $\epsilon=0.109$. The fit results for the lower bound were:

$$
\begin{aligned}
\sigma_{\text {tot }}^{p^{ \pm} p} & =20 s^{0.081}+62 s^{-0.38} \mp 17 s^{-0.42} \\
\sigma_{\text {tot }}^{\pi^{ \pm} p} & =13.32 s^{0.081}+25.91 s^{-0.38} \mp 3.75 s^{-0.42} \\
\sigma_{\text {tot }}^{K^{ \pm} p} & =11.92 s^{0.081}+10.75 s^{-0.38} \mp 6.83 s^{-0.42} \\
\sigma_{\text {tot }}^{\gamma p} & =0.067 s^{0.081}+0.096 s^{-0.38} \\
\sigma_{\text {tot }}^{\gamma \gamma} & =0.00020 s^{0.081}+0.00015 s^{-0.38} .
\end{aligned}
$$

with $\chi^{2} / \mathrm{DOF}=0.91$. With the upper bound we obtained

$$
\begin{aligned}
\sigma_{\text {tot }}^{p^{ \pm} p} & =16 s^{0.109}+51 s^{-0.28} \mp 17 s^{-0.42} \\
\sigma_{\text {tot }}^{\pi^{ \pm} p} & =10.12 s^{0.109}+24.7 s^{-0.28} \mp 3.8 s^{-0.42} \\
\sigma_{\text {tot }}^{K^{ \pm} p} & =9.27 s^{0.109}+13.8 s^{-0.28} \mp 6.9 s^{-0.42} \\
\sigma_{\text {tot }}^{\gamma p} & =0.051 s^{0.109}+0.105 s^{-0.28} \\
\sigma_{\text {tot }}^{\gamma \gamma} & =0.00015 s^{0.109}+0.0002 s^{-0.28} .
\end{aligned}
$$

with $\chi^{2} / \mathrm{DOF}=0.99$.

These parametrizations for the $\pi^{ \pm} p, K^{ \pm} p, \gamma p$ and $\gamma \gamma$ are displayed in Fig. 2 together with the experimental data. We see that, with the exception of the highest energy data points in $\pi^{-} p, \gamma p$ and $\gamma \gamma$ scattering, the experimental data are well described in both cases (upper and lower bounds). We note that the highest points have the largest errors bars and, therefore, little influence in global fits. We shall return to this point in Sec. IV.

The results obtained allow also to check the values of the extrema bounds in terms of the quark counting and factorization rules. From quark counting we expect that

$$
\frac{\sigma_{\pi^{ \pm} p}}{\sigma_{p p}} \sim \frac{2}{3} \approx 0.67 .
$$

Factorization is related with the strength of the Pomeron coupling with the photon and proton and states that

$$
\frac{\sigma_{p p}}{\sigma_{\gamma p}} \sim \frac{\sigma_{\gamma p}}{\sigma_{\gamma \gamma}} .
$$

We check the above rules at the asymptotic region $(\sqrt{s} \rightarrow \infty)$ and since the pomeron contribution is the only that increases with the energy, we neglect the other trajectories.

The results for the ratios from the obtained parameters are shown in Table II. For the quark counting rule, the ratio of the $p p$ to $\pi p$ cross sections agrees better with the expected value of 0.67 for the lower $\epsilon$ value and both results matched the expected ratio when the errors from the fit parameters are taken into account. In the case of the kaon-proton the ratio does not agree with 0.67 in both cases. However, it is not clear if the pomeron coupling with strange quarks have the same strength as to light quarks.

For the factorization relation, Eq. (6), the best agreement comes from the values obtained for the higher $\epsilon$. However, the value obtained for the lower $\epsilon$ is not that far from the expected either, and both results agree when the errors are taken into account.

We conclude that, in the context of the procedure used, the extrema bounds are not in disagreement with both quark counting and factorization rules.

TABLE 2. Results for the quark counting rule and factorization using the pomeron amplitudes obtained from the fits.

\begin{tabular}{ccc}
\hline Extremum: & $\epsilon=0.109$ & $\epsilon=0.081$ \\
\hline$\left(\sigma_{\pi^{ \pm}} / \sigma_{p p}\right) / 0.67$ & $0.92 \pm 0.07$ & $0.97 \pm 0.04$ \\
$\left(\sigma_{K^{ \pm}} / \sigma_{p p}\right) / 0.67$ & $0.84 \pm 0.07$ & $0.87 \pm 0.04$ \\
$\left(\sigma_{p p} / \sigma_{\gamma p}\right) /\left(\sigma_{\gamma p} / \sigma_{\gamma \gamma}\right)$ & $0.96 \pm 0.17$ & $0.90 \pm 0.12$ \\
\hline
\end{tabular}

\section{Conclusions and final remarks}

In this paper we have investigated some effects of the extrema bounds for the soft pomeron intercept in the description of the total cross sections from $\pi^{ \pm} p, K^{ \pm} p, \gamma p$ and $\gamma \gamma$ scattering.

In the context of the global fits, explained in Sec. III, we have shown that both extrems lead to good descriptions of the experimental data, with the exception of the highest data points from $\pi^{-} p, \gamma p$ and $\gamma \gamma$ scattering. Although the extrapolations predict different behaviors for $\sigma_{t o t}(s)$, neither bound are able to describe the above points in a reasonable way, specially in the $\gamma \gamma$ scattering. This last case may suggest the necessity of an additional component, as the hard pomeron. We have also shown that, taking into account the errors in the fit parameters, both bounds do not violate the factorization rule or the quark counting rule (in the case of $p p$ and $\pi^{ \pm} p$ scattering).

Our main conclusion is that, with the procedure used, the data analyzed and the rules tested are not so sensitive to the extrema bounds. We understand that this conclusion brings novel information about the numerical interval that could be associated with a truthful value for the soft pomeron intercept. However, experimental data at higher energies may select the best bound, as suggested by Fig. 2 . 

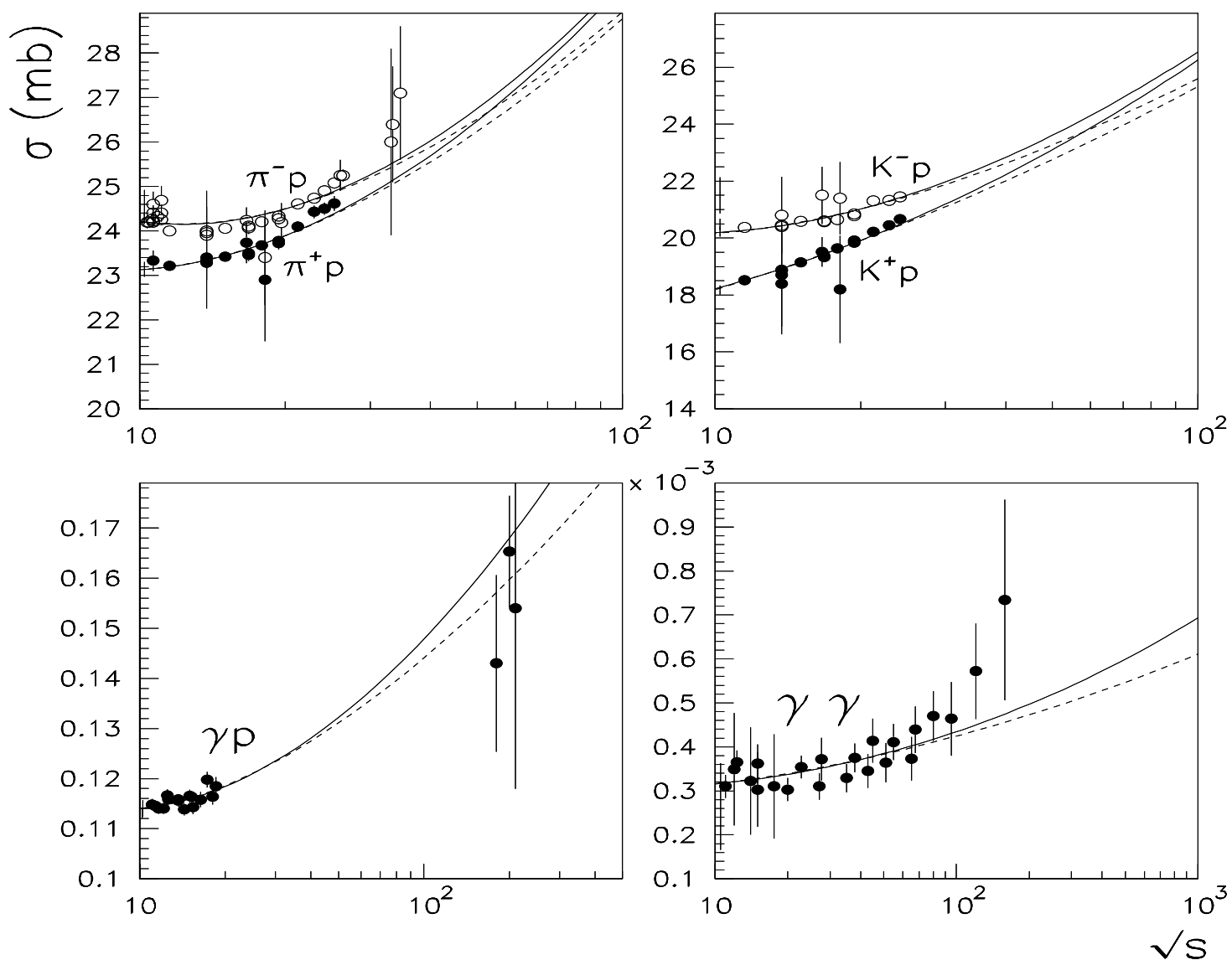

Figure 2. Results of the global fits to pion-proton, kaon-proton, gamma-proton and gamma-gamma total cross section data, with $\epsilon_{u p p e r}=$ 0.109 (solid) and $\epsilon_{\text {lower }}=0.081$ (dashed).

The extensions may also be performed by means of other procedures, as for example, the use of fitted trajectories for the secondary reggeons from the Chew-Frautschi plots. We are, presently, investigating the subject.

\section{Acknowledgments}

We are thankful to FAPESP for financial support (Contract N. 00/00991-7 and 00/04422-7).

\section{References}

[1] Particle Data Group, K. Hagiwara et al., Phys. Rev. D 66, 010001 (2002).

[2] P. D. B. Collins, An Introduction to Regge Theory and High Energy Physics, (Cambridge University Press, Cambridge, England, 1977).
[3] CDF Collaboration, F. Abe et al., Phys. Rev. D 505550 (1993).

[4] E710 Collaboration, N.A. Amos et al., Phys. Rev. Lett. 68 2433 (1992).

[5] E811 Collaboration, C. Avila et al., Phys. Lett. B 445419 (1999); Phys. Lett. B 537, 41 (2002).

[6] R.F. Ávila, E.G.S. Luna, and M.J. Menon, Phys. Rev. D 67, 054020 (2003).

[7] E.G.S. Luna and M.J. Menon, Phys. Lett. B 565, 123 (2003).

[8] N.N. Nikolaev, Phys. Rev. D 48, R1904 (1993).

[9] T.K. Gaisser, U.P. Sukhatme, and G.B. Yodh, Phys. Rev. D 36, 1350 (1987).

[10] M.M. Block, F. Halzen, and T. Stanev, Phys. Rev. Lett. 83, 4926 (1999); Phys. Rev. D 62, 077501 (2000). 